\begin{tabular}{|c|c|}
\hline Title & Nonlinear optical phase shift obtained from two-level atoms confined in a planar microcavity \\
\hline Author(s) & Oka, Hisaki; Fujiwara, Hideki; Takeuchi, Shigeki; Sasaki, Keiji \\
\hline Citation & $\begin{array}{l}\text { Journal of A pplied Physics, 107(5), } 054310 \\
\text { https://doi.org/10.1063/1.3327411 }\end{array}$ \\
\hline Issue Date & 2010-03-01 \\
\hline Doc URL & http:/hdl.handle.net/2115/49853 \\
\hline Rights & $\begin{array}{l}\text { Copyright } 2010 \text { A merican Institute of Phy sics. This article may be downloaded for personal use only. Any other use } \\
\text { requires prior permission of the author and the A merican Institute of Physics. The following article appeared in J. A ppl. } \\
\text { Phys. } 107,054310 \text { (2010) and may be found at https://dx.doi.org/10.1063/1.3327411 }\end{array}$ \\
\hline Type & article \\
\hline File Information & JAP107-5_054310.pdf \\
\hline
\end{tabular}

Instructions for use 


\section{AIP Appilited Physics}

Nonlinear optical phase shift obtained from two-level atoms confined in a planar microcavity

Hisaki Oka, Hideki Fujiwara, Shigeki Takeuchi, and Keiji Sasaki

Citation: J. Appl. Phys. 107, 054310 (2010); doi: 10.1063/1.3327411

View online: http://dx.doi.org/10.1063/1.3327411

View Table of Contents: http://jap.aip.org/resource/1/JAPIAU/v107/i5

Published by the American Institute of Physics.

Additional information on J. Appl. Phys.

Journal Homepage: http://jap.aip.org/

Journal Information: http://jap.aip.org/about/about_the_journal

Top downloads: http://jap.aip.org/features/most_downloaded

Information for Authors: http://jap.aip.org/authors

\section{ADVERTISEMENT}

World's Ultimate AFM Experience the Speed \& Resolution

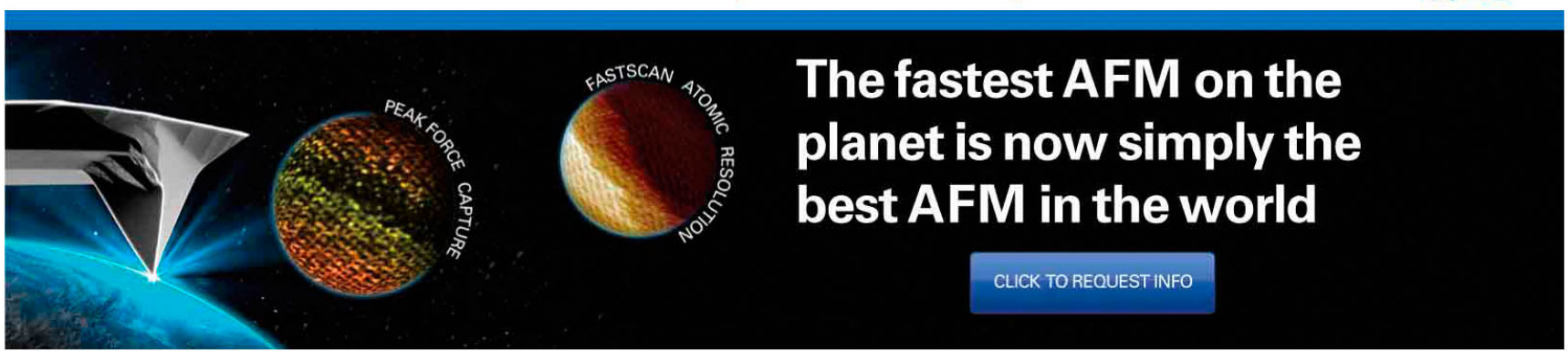




\title{
Nonlinear optical phase shift obtained from two-level atoms confined in a planar microcavity
}

\author{
Hisaki Oka, ${ }^{1, a)}$ Hideki Fujiwara, ${ }^{2}$ Shigeki Takeuchi, ${ }^{2,3}$ and Keiji Sasaki ${ }^{2}$ \\ ${ }_{1}^{1}$ Photon Pioneers Center, Osaka University, Suite Osaka 565-0871, Japan \\ ${ }^{2}$ Research Institute for Electronic Science, Hokkaido University, Sapporo 001-0020, Japan \\ ${ }^{3}$ The Institute for Scientific and Industrial Research, Osaka University, Ibaraki, Osaka 567-0047, Japan
}

(Received 10 October 2009; accepted 20 January 2010; published online 5 March 2010)

\begin{abstract}
We investigate the nonlinear optical phase shift obtained from a thin atomic layer confined in a distributed Bragg reflector (DBR) microcavity with reflection geometry. The optical response of the atom-cavity system is numerically analyzed using finite-difference time-domain method with the optical Bloch equations. The optimal position of atomic layer, at which a maximal phase shift of $\pi$ is realized, drastically changes with the quality factor $Q$ of the cavity. We show that for high $Q$ the maximal phase shift of $\pi$ can be obtained anywhere in the cavity field independently of atomic layer position. This result is in contrast to that obtained from a one-dimensional atom model in the limit of bad cavity, where a maximal phase shift of $\pi$ is obtained only at the antinode of the cavity field. We also show that the independence of phase shift on atom position realized in high- $Q$ regime is due to an interference effect in the surface layers of the DBR cavity. (C) 2010 American Institute of Physics. [doi:10.1063/1.3327411]
\end{abstract}

\section{INTRODUCTION}

The strong optical nonlinearities realizable in cavity quantum electrodynamics (cavity QED) have attracted much attention because of their potential applications in optical quantum information and communication technologies, e.g., in the realization of photonic quantum phase gates (QPG) or quantum controlled-NOT gates. ${ }^{1-4}$ One way of realizing a photonic QPG is to utilize single-atom nonlinearity enhanced by cavity QED effect. ${ }^{2,5}$ In particular, one-dimensional atom model (1D atom) is one of strong candidates for photonic QPG, which can be realized by confining a single atom in a microcavity with reflection geometry so that dipole emission from the atom can be restricted to a $1 \mathrm{D}$ beam profile. ${ }^{6,7}$ The nonlinear optical response of $1 \mathrm{D}$ atom has been theoretically analyzed in terms of the response to one-photon and twophoton input pulses ${ }^{8,9}$ the effects of decoherence on the nonlinear phase shifts, ${ }^{10}$ and optimal cavity QED parameters. ${ }^{11}$ These results indicate that a $1 \mathrm{D}$ atom has a potential not only for a photonic QPG but also for other optical quantum information devices. ${ }^{8,12}$

A scenario to utilize 1D atom as a photonic QPG is summarized as the realization of strong nonlinear photonphoton interaction mediated by a single atom. Since a single atom is fully saturated by one-photon absorption, the atom saturation induces a relative phase shift between two absorbed photons. The final goal is to realize a relative phase shift of $\pi$ between the two photons, being qubits (e.g., their polarizations). This is achieved when $\pi$ phase shift is realized for one-photon input because a subsequent input photon is unaffected by a fully-saturated atom. Although rigorous evaluation of the performance as a photonic QPG requires a fully quantum-mechanical analysis using spatiotemporal

\footnotetext{
a) Author to whom correspondence should be adressed. Electronic mail: oka@ppc.osaka-u.ac.jp.
}

photon wavefunctions, ${ }^{9}$ if we focus only on the amount of phase shift, we can evaluate it by calculating overall nonlinear phase shift using a semiclassical method, ${ }^{6,10}$ which is the total phase shift obtained by varying incident light intensity (mean photon number) from zero to infinity. Since the fully quantum-mechanical analysis is often complicated, the semiclassical approach is quite useful to discriminate whether a target atom-cavity system is suitable for the above QPG scenario using $1 \mathrm{D}$ atom.

Owing to the recent development of semiconductor microcavities, a 1D atom is expected to be realized in lowdimensional semiconductor systems. This could be achieved, e.g., by using a quantum dot in a photonic crystal or a micropost cavity. ${ }^{13}$ However, studies based on $1 \mathrm{D}$ atom could not be directly applied to above systems because cavity fields are adiabatically eliminated by considering the limit of bad cavity; cavity damping rate is much larger than atom-cavity coupling rate. In practice, solid-state microcavities achieve strong light-matter coupling and therefore the bad cavity limit no longer holds true. As a result, cavity-mode profiles and position of atoms within them should be properly considered. In fact, in previous work, we have shown that overall nonlinear phase shift is strongly affected by atom position in an electromagnetic-field profile. ${ }^{14}$ Thus, atom position would be an important parameter characterizing the overall nonlinear phase shift, in particular when a 1D atom is implemented by a solid-state microcavity realizing strong lightmatter coupling.

In this study, we therefore investigate atom-position effects on the overall nonlinear phase shift obtained from a 1D atom beyond the bad cavity limit. Generally, theoretical analyses of the light-matter interaction including atomposition dependence are complicated because calculations of cavity-mode functions are required. Further it is difficult to calculate mode functions of a cavity having a complex struc- 
ture and therefore numerical calculation is necessary. We adopt the finite-difference time-domain (FDTD) method coupled with the optical Bloch equations as used in Refs. 14 and 15. This method has the advantage that we can directly calculate the dynamics of interaction between light and a single atom including atom-position dependence for microcavities with arbitrary shape. As a first step, we analyze the optical response of an atom-cavity system consisting of a thin infinite atomic layer of two-level atoms and a distributed Bragg reflector (DBR) microcavity with reflection geometry. The optical response of this system can be reduced to a 1D problem and corresponds to that of $1 \mathrm{D}$ atom. We will show that optimal position of atomic layer, yielding a overall nonlinear phase shift of $\pi$, varies with cavity quality factor $Q$. In particular, for low- $Q$ cavities, optimal position is not corresponding to the antinode of cavity field, where electric cavity-field strength is maximal, but is instead slightly shifted toward the surface of the cavity. As $Q$ factor increases, however, overall nonlinear phase shift of $\pi$ can be obtained anywhere in the cavity field independently of atomic layer position. This result is contrast to the result obtained in the bad cavity limit, where overall nonlinear phase shift of $\pi$ is obtained at the antinode. ${ }^{14}$ We show that this change in position dependence is due to interference effects in surface DBR layers.

The rest of this paper is organized as follows. In Sec. II, we formulate the dynamics of the Maxwell-Bloch equations in terms of the FDTD method and provide a definition of the overall nonlinear phase shift. In Sec. III, we analyze the effects of the atomic layer position on the overall nonlinear phase shift. In Sec. IV, we summarize our results.

\section{MODEL AND NUMERICAL SETUP}

We consider a DBR microcavity with reflection geometry, as depicted in Fig. 1(a), where the DBR cavity is described in terms of refractive-index distribution. The DBR cavity consists of alternate refractive indices of $n=3.4$ and $n=1$, assuming GaAs and air, respectively. In order to realize a cavity with reflection geometry, the second layer of $n$ $=3.4$ from the left end is removed (an air defect), so that the DBR cavity is formed by left-side and right-side mirrors, consisting of 1 and 20 pairs of layers, respectively. The center of the defect is set to the origin of the $x$-axis. Transmission- and defect-mode spectra are shown in Fig. 1(b). The transmission spectrum (solid line) is obtained for an incident temporal Gaussian pulse with a width of $10 \mathrm{fs}$ and a central frequency of $3.5 \times 10^{14} \mathrm{~Hz}$. At the frequency range from $2.5 \times 10^{14}-3.6 \times 10^{14} \mathrm{~Hz}$, the transmittance is zero (a stop band) and the DBR behaves as a perfect mirror. The defect-mode spectrum (dotted line) is obtained at the origin, where the intensity of the cavity field is maximal. The resultant defect mode is a single monopole and the central frequency is in the stop band. Thus, a single-mode cavity with reflection geometry is realized simply by using a DBR with a near-surface defect. A high- $Q$ microcavity can be achieved by adding a few layers at the left end of the DBR. An atomic layer will be inserted in the defect-cavity region. If the length of the atomic and DBR layers in the $y$ and $z$

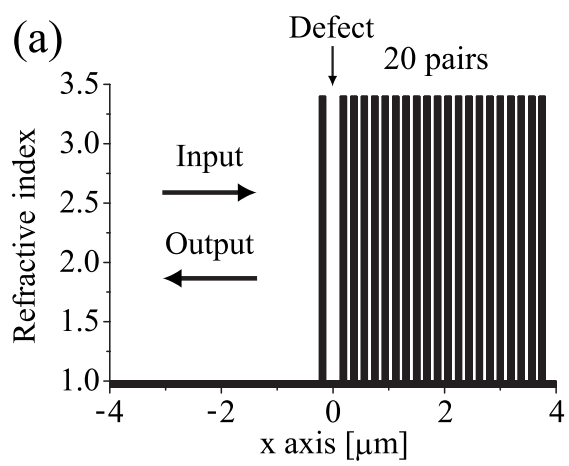

(b)

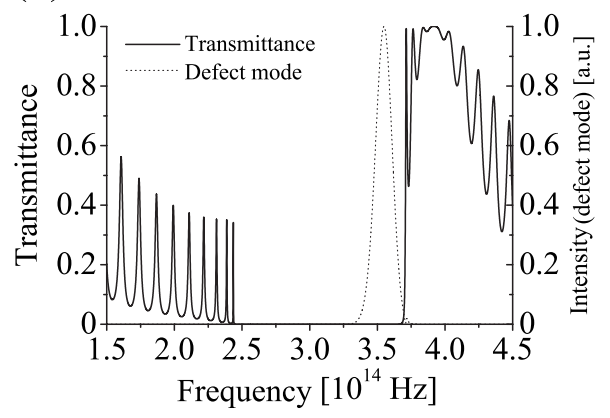

FIG. 1. (a) Refractive-index distribution of a DBR cavity with reflection geometry. (b) Transmission and defect-mode spectra. The incident light is a temporal Gaussian pulse with a width of $10 \mathrm{fs}$ and a central frequency of $3.5 \times 10^{14} \mathrm{~Hz}$.

directions is much larger than the width of the layers, the atom-cavity system can be reduced to a $1 \mathrm{D}$ problem without optical losses, because dipole emission from the atomic layer is restricted to a 1D input-output field. ${ }^{14}$ In terms of the cavity QED parameters, this reduction corresponds to an atom-cavity system with a spontaneous emission factor of $\beta=1$, which is defined as the fraction of spontaneous emission through a specific cavity mode to the total spontaneous emission. ${ }^{13}$

The nonlinear optical response of the atom-cavity system can then be analyzed simply as a 1D Maxwell-Bloch system, where the optical Bloch equations are coupled to the Maxwell's equations through a dipole current. The 1D Maxwell's equations with a dipole current $j_{z}$ are given by

$$
\begin{aligned}
& \frac{\partial E_{z}(x, t)}{\partial t}=\frac{1}{\epsilon(x) \epsilon_{0}}\left[\frac{\partial H_{y}(x, t)}{\partial x}-j_{z}(x, t)\right], \\
& \frac{\partial H_{y}(x, t)}{\partial t}=\frac{1}{\mu_{0}} \frac{\partial E_{z}(x, t)}{\partial x},
\end{aligned}
$$

where $\epsilon(x)$ is the relative dielectric constant. Note that in a 1D system the divergence of the electromagnetic field is always zero because only electromagnetic fields orthogonal to the $x$-axis are allowed. The Bloch equations for a two-level atom with a ground state $|g\rangle$ and an excited state $|e\rangle$ can be expressed in terms of the complex dipole operators, $\hat{\sigma}_{-}$ $=|g\rangle\langle e|$ and $\hat{\sigma}_{+}=|e\rangle\langle g|$, and the inversion operator $\hat{\sigma}_{z}=(|e\rangle$ $\times\langle e|-| g\rangle\langle g|) / 2$ as $^{16}$

$$
\frac{d}{d t}\left\langle\hat{\sigma}_{-}\right\rangle=\left(-\gamma_{\|}-i \omega_{0}\right)\left\langle\hat{\sigma}_{-}\right\rangle-i 2 \Omega\left\langle\hat{\sigma}_{z}\right\rangle,
$$




$$
\begin{aligned}
& \frac{d}{d t}\left\langle\hat{\sigma}_{+}\right\rangle=\left(-\gamma_{\|}+i \omega_{0}\right)\left\langle\hat{\sigma}_{+}\right\rangle+i 2 \Omega\left\langle\hat{\sigma}_{z}\right\rangle, \\
& \frac{d}{d t}\left\langle\hat{\sigma}_{z}\right\rangle=-2 \gamma_{\|}\left(\left\langle\hat{\sigma}_{z}\right\rangle+\frac{1}{2}\right)-i \Omega\left(\left\langle\hat{\sigma}_{-}\right\rangle-\left\langle\hat{\sigma}_{+}\right\rangle\right),
\end{aligned}
$$

where $\gamma_{\|}$is the dipole decay rate due to spontaneous emission, $\omega_{0}$ is the resonant frequency between the two atomic levels, and $\Omega$ is the Rabi frequency given by

$$
\Omega=\frac{d_{\mathrm{eg}} E_{z}\left(x_{a}, t\right)}{\hbar},
$$

where $d_{\text {eg }}$ is the dipole moment of the atom and $x_{a}$ denotes the position of the atomic layer. Since the dipole current arises from the induced polarization $P_{z}$ of the atoms, it can be expressed in terms of the polarization dynamics. Using the density of polarized atoms in the atomic layer, $N_{\text {atom }}$, the dipole current $j_{z}$ can be described as

$$
j_{z}(x, t)=N_{\text {atom }} d_{\mathrm{eg}} \delta\left(x-x_{a}\right) \frac{d}{d t}\left(\left\langle\hat{\sigma}_{-}\right\rangle+\left\langle\hat{\sigma}_{+}\right\rangle\right),
$$

showing how the coherent dipole emission from the twolevel atoms in the atomic layer is coupled to Maxwell's equations through the dipole current $j_{z}$. If $N_{\text {atom }}$ is chosen so that one polarized atom is in each volume enclosed by the atomic cross section $A$ of a single atom and the thickness $\ell$ of the atomic layer,

$$
N_{\text {atom }}=\frac{1}{A \ell} \quad \text { with } \quad A=\frac{2 \pi c^{2}}{\omega_{0}^{2}},
$$

equation (4) corresponds to dipole emission from a single atom. The optical response of the 1D Maxwell-Bloch system can thus be reduced to that of a $1 \mathrm{D}$ atom with no optical losses.

The overall nonlinear phase shift $\Delta \phi$ is defined as the total change of the phase difference $\phi\left(\bar{n}_{\text {in }}\right)$ obtained by varying input photons $\bar{n}_{\text {in }}$ from zero to infinity 6,10

$$
\Delta \phi=\lim _{\bar{n}_{\text {in }} \rightarrow \infty} \phi\left(\bar{n}_{\text {in }}\right)-\lim _{\bar{n}_{\text {in }} \rightarrow 0} \phi\left(\bar{n}_{\text {in }}\right),
$$

where $\phi\left(\bar{n}_{\text {in }}\right)$ is the phase difference between the input and output photons at $\bar{n}_{\text {in }}$, with reference to that obtained from an empty cavity; $\phi\left(\bar{n}_{\text {in }}\right)=0$ means the response of an empty cavity. $\bar{n}_{\text {in }}$ is the time-averaged input photons defined as $\bar{n}_{\text {in }}$ $=\bar{S} A / \hbar \omega \gamma_{\|}$, where $\bar{S}$ is the time-averaged Poynting vector component given by $\bar{S}=\overline{E_{z} H_{y}}$. In the limit of high-intensity input field $\left(\bar{n}_{\text {in }} \rightarrow \infty\right)$, the atoms are completely saturated and the inversion is $\left\langle\hat{\sigma}_{z}\right\rangle_{s}=0$. The saturation of $\left\langle\hat{\sigma}_{z}\right\rangle_{s}$ also affects the atomic dipole, so that $\left\langle\hat{\sigma}_{-}\right\rangle_{s}$ goes to zero and the optical response of the atom-cavity system is the same as that of an empty cavity. As a result, $\phi(\infty)=0$ and $\Delta \phi$ is therefore equal to the negative phase difference obtained for intensities close to zero, that is, $\Delta \phi=-\phi(0)$. When an atom-cavity system with $\beta>1 / 2$ is realized, a maximal phase shift of $\pi$, i.e., $|\Delta \phi|=\pi$, can be achieved. ${ }^{6,10}$

The Maxwell-Bloch Eqs. (1) and (2) can be numerically solved by transforming them into spatiotemporal finitedifference equations and by using the FDTD method coupled

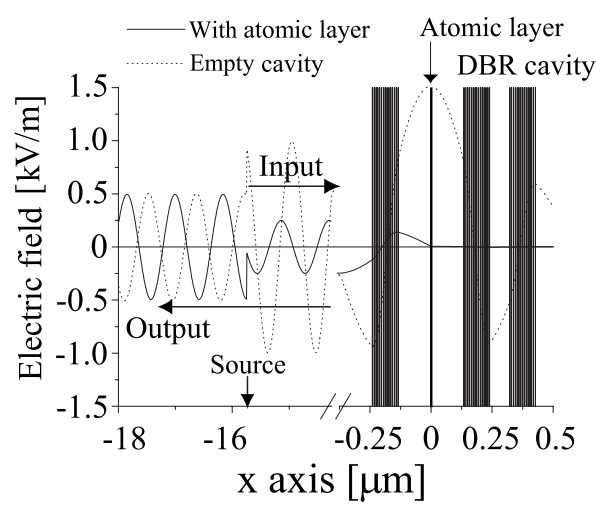

FIG. 2. Snapshot of the electric-field profile for $\bar{n}_{\text {in }} \approx 0.0036$ at $t$ $=0.1247615 \mathrm{~ns}$. The atomic layer is located at the antinode of the cavity field. In order to clearly show the electric field at the atomic layer and the output field, we omit the range from -14 to -0.375 on the $x$-axis.

to the optical Bloch equations (see Ref. 14 for details). In the following FDTD calculation, we use a cell size of $\Delta x=7.5$ $\times 10^{-9} \mathrm{~m}$ and a time step of $\Delta t \approx 2.499 \times 10^{-17} \mathrm{~s}$. For the DBR cavity parameters, the relative dielectric constant is $\epsilon(x)=11.56$, corresponding to a refractive index of $n=3.4$. The lattice constant of DBR and the layer width are 25 and 15 cells, respectively. Three DBR cavities of different $Q$ are used, where the $Q$ values are obtained by changing the number of left-sided DBR layers between 1, 3, and 5. An example for one layer is shown in Fig. 1, where the central frequency of the defect-cavity mode is $\omega_{c} / 2 \pi \simeq 3.564$ $\times 10^{14} \mathrm{~Hz}$ and the full width at half maximum is 1.5 $\times 10^{13} \mathrm{~Hz}$, corresponding to $Q=24$. The resonant transition frequency of atoms is the same as the frequency of the defect-cavity mode, $\omega_{0}=\omega_{c}$, and the density of polarized atoms is $N_{\text {atom }}=1.172 \times 10^{21} \mathrm{~m}^{-3}$. For the purpose of shortening computational time, the dipole moment is set to a large value, $d_{\mathrm{eg}}=8.0 \times 10^{-28} \mathrm{C} \mathrm{m}$. The spontaneous emission rate is calculated from

$$
2 \gamma_{\|}=\frac{\left|d_{\mathrm{eg}}\right|^{2} \omega_{0}^{3}}{\pi \epsilon_{0} \hbar c^{3}}
$$

where it is assumed that all the atoms in the atomic layer are oriented in the same direction as the polarization of input field $\left(\gamma_{\|} / 2 \pi \approx 10^{10} \mathrm{~Hz}\right.$ for the present parameters $)$. The thickness of atomic layer is $\ell=\Delta x$ (i.e., one cell). For $\bar{n}_{\text {in }}$ $\ll 1, \Delta \phi$ can be obtained by comparing the output from an atomic layer to that from an empty cavity. ${ }^{17}$ Throughout this work, we use $\bar{n}_{\text {in }} \simeq 0.0036$, corresponding to $\Omega / 2 \pi$ $\approx 10^{9} \mathrm{~Hz}$, so that the system is always in the weak coupling regime $\left(\kappa=\omega_{c} / Q>\gamma_{\|}>\Omega\right)$.

\section{NUMERICAL RESULTS}

In this section, we numerically analyze the nonlinear optical phase shift obtained from an atomic layer coupled to a defect-cavity field. First, we analyze the dependence of overall nonlinear phase shift on the atomic layer position for low- $Q$ and high- $Q$ DBR cavities in the weak coupling regime. Then, we discuss in detail the atom-position dependence for high- $Q$ cavities.

Figure 2 shows a snapshot of electric-field profile calcu- 


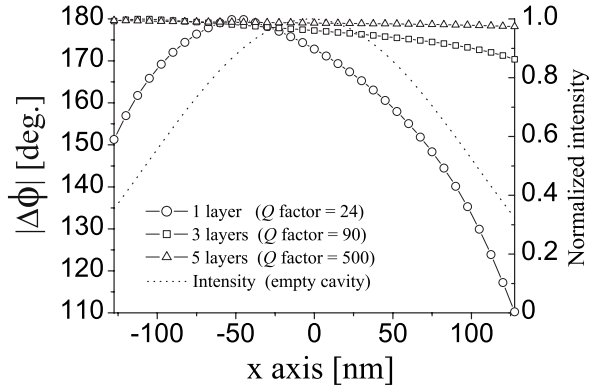

FIG. 3. $|\Delta \phi|$ as a function of the atomic layer position for three DBR cavities with $Q=24,90$, and 500 . The dotted curve is the normalized electric-field strength.

lated by 1D FDTD method, where an atomic layer is placed at $x=0$, corresponding to the antinode of a defect-cavity mode. A rightward traveling continuous wave resonant with the defect-cavity mode is emitted from an input source (indicated by arrow) and is incident on the atom-cavity system. Output from the atom-cavity system is obtained on the left of the input source, where the output consists only of the light reflected by the atom-cavity system. ${ }^{18}$ In order to eliminate back reflections into the computational domain, we use Mur's first-order absorbing boundary condition at both ends of the computational domain. By comparing the output from the atomic layer (solid line) to that for an empty cavity (dotted line), one can find that they are almost in antiphase, $|\Delta \phi| \simeq \pi$. This phase shift occurs owing to interference between the input field and coherent dipole emission from the atomic layer. For $n_{\text {in }} \ll 1$ on resonance, dipole emission from the atomic layer is in antiphase with a local electric field at the atomic layer position, ${ }^{19}$ and the electric field for $x>0$ is therefore attenuated by destructive interference between the input light and the emitted light from the atomic layer toward $x>0$. This effect can be seen for $x>0$ in Fig. 2. As a result, only the reflected component consisting of the light emitted from the atomic layer toward $x<0$, can be measured in the output field. ${ }^{20}$ Thus, the atomic layer acts as a perfect mirror. This leads to optical path difference from an empty cavity, and the phase shift corresponding to the optical path difference, e.g., $|\Delta \phi| \simeq \pi$ at the antinode, can be observed. ${ }^{14}$ Therefore $|\Delta \phi|$ is expected to be strongly affected by atomic layer position.

Figure 3 shows $|\Delta \phi|$ as a function of atomic layer position for three $Q$ factors. The dotted curve is the normalized electric-field strength. For low $Q$ of $Q=24,|\Delta \phi|$ has a similar atom-position dependence to that in the bad cavity limit. However, optimal position yielding $|\Delta \phi|=\pi$ is not at the antinode of the cavity field but is instead slightly shifted toward the surface of the cavity ( $\sim 50 \mathrm{~nm}$ for present parameters). As $Q$ increases to $Q=90$, the values of $|\Delta \phi|$ are elevated overall and the optimal position further shifts toward the surface. For further increase to $Q=500,|\Delta \phi| \simeq \pi$ can be obtained anywhere independently of the atomic layer position.

Thus, $\Delta \phi$ depends on atomic layer position and this dependence changes with increase in $Q$ factor. Figure 4 shows the electric-field profiles of 1-surface-layer and 5-surfacelayer DBR cavities for four different positions of atomic
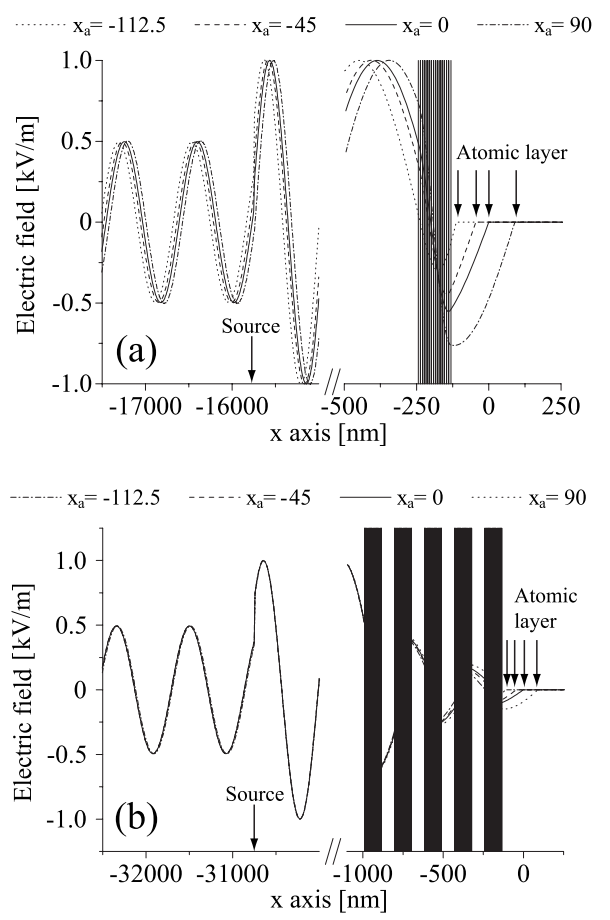

FIG. 4. Electric-field profiles of (a) a 1-layer DBR cavity and (b) a 5-layer DBR cavity for four different positions of the atomic layer.

layer, $x_{a}=-112.5,-45,0$, and $90 \mathrm{~nm}$. The electric fields for $x>x_{a}$ are reduced to almost zero owing to destructive interference, and thus the atomic layer acts as a perfect mirror. For the 1-surface-layer DBR cavity, this process is almost the same as the bad-cavity-limit model, except that the dipole emission is slightly modified by the surface DBR layer. According to the bad-cavity-limit model, a maximal phase shift of $\pi$ is obtained when optical path difference between the outputs for an empty cavity and for the atomic layer is equal to half a wavelength. In particular, in the bad cavity limit, it occurs when the atomic layer is at the antinode of electric field $(x=0)$. However, when the surface DBR layer is present, multiple reflection of light between the surface DBR layer and the atomic layer modifies output phase, and this leads to a shift in optimal position. In fact, in the absence of surface DBR layer, no shift in optimal position occurs and the maximal phase shift of $\pi$ is obtained at the antinode of electric field as in the bad cavity limit. Thus, for low- $Q$ cavities, the multiple reflection induces only a shift in optimal position.

For a 5-surface-layer DBR cavity, however, phase difference due to change in $x_{a}$ almost disappears. As the number of surface layer increases, reflectance of the surface DBR layers increases. As a result, in contrast to 1-surface-layer DBR, two standing waves are formed between the surface DBR and the atomic layer and between the atomic layer and the back-surface DBR so that the atomic layer acts as a node for these standing waves. This leads to amplitude attenuation of the latter standing wave, especially for high- $Q$ cavities. Furthermore, the optical waves penetrating the surface DBR become in phase owing to phase matching by multiple reflection in the surface DBR, as can be seen in Fig. 4(b). Once the atomic layer acts as a node (like a perfect mirror), the backsurface DBR has no effect on $\Delta \phi$. Instead a new mechanism 
(a)

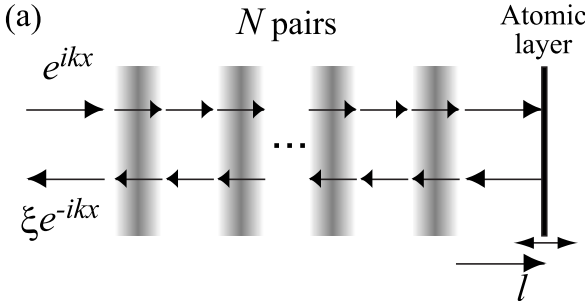

(b)

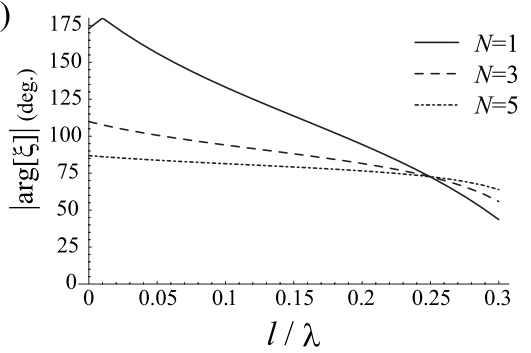

FIG. 5. (a) Schematic of multiple reflection approximation with a fixed end. (b) Dependence of output phase $|\arg [\xi]|$ on $l$ for $N=1,3$, and 5 .

for altering $\Delta \phi$ appears, governed by the surface DBR. This can be explained by using a simple multiple reflection problem with a fixed end, as depicted in Fig. 5(a), where $\xi$ is the complex amplitude of output field and an atomic layer is assumed to be a perfect mirror. Figure 5(b) shows dependence of the output phase $|\arg [\xi]|$ on atom position $l$ for three $N$-surface-layer DBRs, $N=1,3$, and 5. For $N=1,|\arg [\xi]|$ widely changes in $l$ and has a local maximum. As $N$ increases, however, this local maximum disappears and dependence of $|\arg [\xi]|$ on $l$ becomes gradual, similar to the dependence of $|\Delta \phi|$. Note that the value of $|\arg [\xi]|$ is not equal to $|\Delta \phi|$ because $\Delta \phi$ is defined with reference to the phase shift obtained from an empty cavity. The position independence of $\Delta \phi$ is thus specific to high- $Q$ cavities.

The realization of $|\Delta \phi|=\pi$ independent of $x_{a}$ is helpful for device applications because high-accuracy positioning of atoms within a microcavity is not that easy. Note, however, that the results obtained in the present work are for a monopole mode (a $\lambda / 2$ cavity). In contrast, a $\lambda$ cavity has a node within the cavity field. If one misaligns the atomic layer position near a node, the transmission component (for $x>0$ ) does not completely vanish and the overall nonlinear phase shift drastically decreases. One then needs to take into account for reabsorption and re-emission of atoms due to the transmission component. ${ }^{14}$ In general, the reabsorption-reemission process is complicated because the re-emission component is reflected by the cavity mirror, inducing subsequent re-emission. Consequently, the analysis of the nonlinear phase shift for a $\lambda$ cavity is rather involved. However, the FDTD analysis would be useful for analyzing such processes in the presence of a surface interference effect, even for systems with a complex device structure.

\section{CONCLUSIONS AND DISCUSSION}

Using the FDTD method with the optical Bloch equations, we have investigated the nonlinear optical phase shift obtained from a thin, infinite atomic layer of two-level atoms confined in a DBR cavity with reflection geometry. We have shown that the optimal position of the atomic layer, yielding a maximal phase shift of $\pi$, varies according to the quality factor $Q$ of the cavity. For low- $Q$ cavities, the optimal position is not at the antinode of the cavity field but is instead slightly shifted toward the surface of the DBR cavity. As the $Q$ value increases, however, a maximal phase shift of $\pi$ can be obtained anywhere in the cavity field independently of atomic layer position. This result is in contrast to the result for the bad cavity limit, for which the maximal phase shift of $\pi$ is obtained only at the antinode. We have also shown that the change in the atom-position dependence for $Q$ factor is due to interference effects in the surface of the DBR cavity. In particular, for high- $Q$ cavities the surface interference compensates the phase lag due to the atomic layer position.

In this analysis, we have investigated the nonlinear optical phase shift for a coherent $\mathrm{cw}$ input field and have restricted the calculation to the atom-cavity system with $\beta=1$ in order to compare the results with the previous work. However, it is straightforward to extend the analysis to a system driven by pulsed input lights. It would be interesting to consider the two-photon nonlinearity discussed in Ref. 21. For atom-cavity systems with $\beta<1$, we can analyze the optical phase shift by introducing a dipole current multiplied by $\beta$, $\beta j_{z}(x, t)$, in Eq. (1). In this case, according to Refs. 6 and 10 , a maximal phase shift of $\pi$ is obtained only for $\beta>1 / 2$. For $\beta<1 / 2$, the maximal phase shift cannot exceed $\pi / 2$.

Finally, FDTD calculation is useful for evaluating the performance of optical cavities with higher dimensions and more complicated structures. We hope that the results presented in this paper help to identify practical requirements for implementing efficient nonlinear devices.

\section{ACKNOWLEDGMENTS}

We thank Dr. H. F. Hofmann for helpful advice. This work was supported by the Japan Science and Technology Agency (JST), Ministry of Internal Affairs and Communication (MIC), Japan Society for the Promotion of Science (JSPS), 21st Century COE Program, Special Coordination Funds for Promoting Science and Technology.

${ }^{1}$ M. A. Nielsen and I. L. Chuang, Quantum Computation and Quantum Information (Cambridge University Press, Cambridge, 2000).

${ }^{2}$ Q. A. Turchette, C. J. Hood, W. Lange, H. Mabuchi, and H. J. Kimble, Phys. Rev. Lett. 75, 4710 (1995).

${ }^{3}$ A. Rauschenbeutel, G. Nogues, S. Osnaghi, P. Bertet, M. Brune, J. M. Raimond, and S. Haroche, Phys. Rev. Lett. 83, 5166 (1999).

${ }^{4}$ A. Imamoglu, D. D. Awschalom, G. Burkard, D. P. DiVincenzo, D. Loss, M. Sherwin, and A. Small, Phys. Rev. Lett. 83, 4204 (1999).

${ }^{5}$ M. Veith, K. G. Müller, S. Mittler-Neher, and W. Knoll, Appl. Phys. B: Lasers Opt. 60, S1 (1995).

${ }^{6}$ H. F. Hofmann, K. Kojima, S. Takeuchi, and K. Sasaki, J. Opt. B: Quantum Semiclassical Opt. 5, 218 (2003).

${ }^{7}$ K. Kojima, H. F. Hofmann, S. Takeuchi, and K. Sasaki, Phys. Rev. A 68, 013803 (2003).

${ }^{8}$ H. F. Hofmann, K. Kojima, S. Takeuchi, and K. Sasaki, Phys. Rev. A 68, 043813 (2003).

${ }^{9}$ K. Kojima, H. F. Hofmann, S. Takeuchi, and K. Sasaki, Phys. Rev. A 70, 013810 (2004).

${ }^{10}$ H. Oka, H. F. Hofmann, S. Takeuchi, and K. Sasaki, Jpn. J. Appl. Phys., Part 1 43, 7495 (2004).

${ }^{11}$ K. Koshino and H. Ishihara, Phys. Rev. A 70, 013806 (2004).

${ }^{12}$ K. Kojima and A. Tomita, Phys. Rev. B 73, 195312 (2006).

${ }^{13}$ Y. Yamamoto, S. Machida, and G. Bjork, Phys. Rev. A 44, 657 (1991). 
${ }^{14}$ H. Oka, S. Takeuchi, and K. Sasaki, Phys. Rev. A 72, 013816 (2005).

${ }^{15}$ R. W. Ziolkowski, J. M. Arnold, and D. M. Gogny, Phys. Rev. A 52, 3082 (1995).

${ }^{16}$ D. F. Walls and G. J. Milburn, Quantum Optics (Springer, Berlin, 1995). ${ }^{17}$ J. W. Noh, A. Fougeres, and L. Mandel, Phys. Rev. A 45, 424 (1992).

${ }^{18}$ Note that the notch appearing at the input source is not due to numerical errors but is due to a phase lag between the output field of traveling wave and the standing wave formed by the reflected light and the input field. Such a notch is therefore generally observed in the systems with the reflection geometry that we consider.

${ }^{19}$ A. V. Durrant, Am. J. Phys. 44, 630 (1976).

${ }^{20}$ Note that, for $\mathrm{cw}$ excitation, this destructive interference effect is realized when the atoms in the atomic layer are in a steady state.

${ }^{21}$ K. Koshino and H. Ishihara, Phys. Rev. Lett. 93, 173601 (2004). 